\title{
ADAM15 is involved in MICB shedding and mediates the effects of gemcitabine on MICB shedding in $\mathrm{PANC}-1$ pancreatic cancer cells
}

\author{
XIAOHUI DUAN $^{1}$, XIANHAI MAO $^{1}$ and WEIJIA SUN ${ }^{2}$ \\ ${ }^{1}$ Department of Hepatobiliary Surgery, Hunan Provincial People's Hospital, Changsha, Hunan 410005; \\ ${ }^{2}$ Department of Gerneral Surgery, Xiangya Hospital, Central South University, Changsha, Hunan 410008, P.R. China
}

Received September 16, 2012; Accepted December 5, 2012

DOI: $10.3892 / \mathrm{mmr} .2013 .1272$

\begin{abstract}
The aim of this study was to investigate the role of ADAM15 in MHC class I polypeptide-related sequence B (MICB) protein ectodomain shedding and observe whether or not gemcitabine affects MICB shedding from PANC-1 cells. In this study, immunohistochemistry of MICB and ADAM15 were performed on tumor samples obtained from 93 patients with pancreatic ductal adenocarcinoma (PDAC). The expression of MICB and ADAM15 in the PDAC tissues was significantly higher compared with that in the normal tissues of the pancreas. Statistical analysis showed a significant correlation between the expression of MICB and certain classic clinicopathological characteristics (i.e., histological grade and TNM stage). ADAM15 expression was found to correlate with lymph node metastasis and TNM stage. The Spearman's rank test suggested that the expression of MICB was inversely correlated with that of ADAM15 in PDAC tissues. Knockdown of ADAM15 in PANC-1 cells clearly upregulated MICB expression on the cellular surface and downregulated soluble MICB (sMICB) levels in the culture supernatants. A nontoxic dose of $0.5 \mu \mathrm{mol} / 1$ gemcitabine suppresses ADAM15 expression leading, at the same time, to an increase in MICB expression and a decrease in SMICB production in PANC-1 cells. The mRNA levels of MICB did not change following PANC-1 exposure to gemcitabine. Further study suggests that the suppressive effect of gemcitabine on MICB shedding in PANC-1 cells is mediated by ADAM15 downregulation. In conclusion, the results of the present study support the hypothesis that ADAM15 is involved in MICB shedding of PANC-1 cells and that gemcitabine inhibits MICB ectodomain shedding through the suppression of ADAM15.
\end{abstract}

Correspondence to: Dr Xianhai Mao, Department of Hepatobiliary Surgery, Hunan Provincial People's Hospital, 61 West Liberation Road, Changsha, Hunan 410005, P.R. China

E-mail: maoxianhai@yeah.net

Key words: pancreatic cancer, PANC-1 cells, MHC class I polypeptide-related sequence B, ADAM15, gemcitabine

\section{Introduction}

Pancreatic cancer remains a significant, unresolved therapeutic challenge with similar incidence and mortality rates $(1,2)$. Complete resection remains the only therapeutic option. Thus, the overall 5-year survival of the patients has not been improved over the last 2 decades $(3,4)$. Therefore, it is necessary to develop novel therapeutic approaches to treat pancreatic cancer. Over the past decade, improved understanding and knowledge of the immune system have generated novel strategies for immunotherapy (5). While the success of tumor immunotherapy primarily relies on the identification of tumor antigens, the expression of transformation-associated stress genes commonly provokes innate immune reactions. These responses may be exploited to develop immunotherapeutic approaches to treat cancer (6).

MHC class I polypeptide-related sequence B (MICB) is a glycosylated, polymorphic and membrane-anchored non-classical MHC class I molecule, and is a stress-induced antigen. In normal tissue, MICB has been shown to be mostly restricted to the gastrointestinal tract, but was also shown to be stress inducible in a series of cell lines. However, MICB is upregulated by a range of primary tumors, including lung, kidney, prostate, breast and colon cancers (7). Immune response induced by MICB-NKG2D has been well documented to play an important role in the eradiation of tumors by $\mathrm{T}$ and/or NK cells $(8,9)$.

A previous study suggested that MICB was induced and expressed widely in pancreatic cancer cell lines (10). However, MICB can be cleaved by proteolytic shedding and released into the bloodstream as soluble MICB (sMICB). sMICB has been correlated with poor differentiation and high tumor stage of pancreatic cancer (11). Two mechanisms have been proposed, whereby release of $\mathrm{SMICB}$ may reduce immunogenicity of tumor cells: the shedding of MICB leads to decreased MICB expression levels on the tumor cell surface, which directly affects tumor cell lysis (12). Secondly, it is reported that sMICB shedding from membrane-binding MICB on tumor cells impaired NKG2D-mediated cytotoxicity by reducing the expression of NKG2D receptor through internalization and degradation (13). Therefore, elucidating the molecular mechanisms of MICB shedding from the pancreatic cancer cell membrane and, subsequently, developing therapeutic 
strategies to suppress MICB shedding may be of substantial benefit for pancreatic cancer treatment.

Previously, members of the metzincin superfamily, such as ADAMs (a disintegrin and metalloproteinase), have been reported to play crucial roles in the proteolytic release of the ectodomain of transmembranous proteins, including MICB, from the cell surface (14). MICB shedding of CV1 cells and human glioma U373 cells was found to be inhibited through the silencing of the ADAM17 proteases. This suggests that ADAM17 is involved in the shedding of membrane-bound MICB (15). However, it remains to be determined wherther other ADAM proteases are able to affect MICB shedding.

Gemcitabine is a first-line chemotherapy drug for pancreatic cancer. Gemcitabine alone or in combination with 5-fluorouracil or radiation treatment may prolong the survival of pancreatic cancer patients (16). Plate et al (17) characterized the change of immune cells in pancreatic cancer patients treated with gemcitabine. The data suggest that gemcitabine therapy may increase memory $\mathrm{T}$ cells and promote naïve $\mathrm{T}$-cell activation, and that gemcitabine therapy is not immunosuppressive, but may enhance antitumor immunity induced by tumor vaccine. To develop further uses for gemcitabine in pancreatic cancer treatment, its immunological impact needs to be evaluated.

In the present study, we investigated the expression of MICB and ADAM15 in pancreatic ductal adenocarcinoma (PDAC), and studied the correlation between the clinicopathological characteristics and the expression of MICB and ADAM15, further showing the relevance of MICB and ADAM15 in PDAC. Of note, ADAM15 knockdown experiments demonstrated the essential roles of ADAM15 protease in the shedding of MICB molecules. Gemcitabine, a nucleoside analog that has been approved as an antipancreatic cancer molecularly targeted chemotherapy, effectively downregulated sMICB while upregulating membrane-bound MICB via inhibition of ADAM15.

\section{Materials and methods}

Subjects and samples. Ninety-three patients with PDAC [56 males and 37 females; age, 40-83 years (mean age, 67.3 years)] were enrolled between January 2004 and June 2009. The patients were surgically treated in the Xiangya Hospital affiliated to the Central South University (Hunan, China). Fifteen normal pancreatic tissues were collected through an organ donor procurement program, whenever there was no suitable recipient for pancreas transplantation. The samples of cancer tissue and normal pancreatic tissues were obtained during surgery. The samples were then fixed in $10 \%$ formalin solution and embedded in paraffin. The diagnosis of the samples was confirmed histopathologically. The use of the clinical samples for analysis was approved by the Ethics Committee of the Central South University.

Immunohistochemistry. Immunohistochemical staining was performed using the streptavidin-biotin peroxidase method as previously described (18). MICB monoclonal antibodies (sc-80527, dilution 1:100; Santa Cruz Biotechnology, Inc., Santa Cruz, CA, USA) and mouse anti-ADAM15 monoclonal antibodies (sc-365752, dilution 1:100; Santa Cruz Biotechnology, Inc.) were used in this study.
Evaluation of MICB and ADAM15 immunohistochemical staining. Two independent investigators unaware of patient outcomes assessed the sections. This was a double-blind study. MICB expression was evaluated according to staining intensity and scored (18): negative (-), no staining in cancer cells (same or weaker compared with the cancer stroma); weak expression (+), the staining of the cancer cells is a little stronger compared with that of the cancer stroma in the whole area, or much stronger in a limited $(<20 \%)$ area; strong expression $(++)$, the staining of the cancer cells is much stronger compared with that of the cancer stroma in the whole section. In these groups, the weak (+) and strong (++) cases were considered as positive results for statistical analysis. Cases with negative (-) and weak expression $(+)$ were defined as the low-expression group, and cases with strong expression (++) were defined as the high-expression group.

The total immunohistochemical staining score for ADAM15 staining of each section was calculated according to the percentage of positive-staining tumor cells and the staining intensity (19). The percentage of positive cells was judged ranging from 1 to $4(1,<10 \% ; 2,10-50 \% ; 3,51-80 \% ; 4,>80 \%$ positive tumor cells), while the level of staining intensity was classified between 0 and 3 ( 0 , negative; 1 , weak; 2 , moderate; 3 , strong staining). Multiplying positive-cell numbers by staining intensity yielded a score of $0-12$. We considered samples as positive where the score was $>2$. A score of 3-6 was regarded as moderate $(+)$ and $>6$ as strong $(++)$.

Tumor cell lines and culture. The PANC-1 cell line was previously established in our laboratory. The cells were cultured in RPMI-1640 medium (Sigma-Aldrich, St. Louis, MO, USA) supplemented with $10 \%$ fetal bovine serum (FBS; Sigma-Aldrich), $100 \mathrm{U} / \mathrm{ml}$ penicillin and $100 \mu \mathrm{g} / \mathrm{ml}$ streptomycin, and were maintained at $37^{\circ} \mathrm{C}$ with $5 \% \mathrm{CO}_{2}$ in a humidified atmosphere. The experiments were carried out on logarithmically growing cells.

RNA silencing. The small interfering RNA (siRNA) method was used to knockdown ADAM15. Stealth RNAi oligonucleotide targeting ADAM15 and scrambled oligonucleotides as the control were purchased from Invitrogen (Carlsbad, CA, USA). Cells were transfected by RNAiMAX Transfection reagent (Invitrogen) with $50 \mathrm{nmol} / 1$ siRNA. The siRNAs used were: ADAM15, 5'-AACCCAGCTGTCACCCTCGAA-3'; scramble control, 5'-TTCGAGGGTGACAGCTGGGTT-3'.

Real-time reverse-transcription polymerase chain reaction (RT-PCR). Total RNA was extracted using TRIzol RNA extraction reagent (Sigma-Aldrich), according to the manufacturer's instructions. cDNA was synthesized with the SuperScript III First-Strand Synthesis kit (Invitrogen) and kept frozen at $-20^{\circ} \mathrm{C}$ until analysis. cDNA was amplified (40 cycles, $95^{\circ} \mathrm{C}$ for $15 \mathrm{sec}, 60^{\circ} \mathrm{C}$ for $\left.1 \mathrm{~min}\right)$ using ABI PRISM ${ }^{\circledR} 7700$ Sequence Detection system (Applied Biosystems, Foster City, CA, USA) in a final volume of $20 \mu \mathrm{l}$ containing $1 \mu \mathrm{l}$ of cDNA, $12.5 \mu \mathrm{l}$ of SYBR-Green Master mix (Applied Biosystems) and $0.5 \mu \mathrm{M}$ forward and reverse primers in DNAse-free water. The $\Delta \mathrm{Ct}$ method was used for relative quantifications. The primer sequences used in this study were: ADAM15 forward, 5'-AGC CTCAAAAAGGTGCTTCA-3' and reverse, 5'-CCCTGGTAG 
CAGCAGTTCTC-3'; MICB forward, 5'-CTTCGTTACAAC CTCATGGT-3' and reverse, 5'-ATATGAGTCAGGGTCCTC CT-3'; 18S rRNA forward, 5'-CCATCCAATCGGTAGTAG CG-3' and reverse, 5'-GTAACCCGTTGAACCCCATT-3'.

Western blotting. Western blot analysis was carried out as previously described (20). PANC-1 cells were lysed by the addition of lysis buffer containing $0.5 \%$ sodium deoxycholate, $1 \%$ Nonidet P-40, $50 \mathrm{mM}$ Tris- $\mathrm{HCl}$ and $150 \mathrm{mM} \mathrm{NaCl}$. Cell lysates were cleared by centrifugation at $16,000 \mathrm{rpm}, 4^{\circ} \mathrm{C}$ for $10 \mathrm{~min}$. Cleared lysates were boiled for $5 \mathrm{~min}$ at $100^{\circ} \mathrm{C}$ after the addition of 5X sample loading buffer containing $1 \mathrm{M}$ Tris- $\mathrm{HCl}$, sodium dodesylsulphate, glycerol and bromphenolblue. The samples were electrophoresed at $200 \mathrm{~V}$ on $12.5 \%$ polyacrylamide gels and transferred to nitrocellulose membranes (Bio-Rad, Hercules, CA, USA), blocked with 5\% non-fat dry milk and incubated with primary antibody. Anti-ADAM15 (sc-365752, dilution 1:1,000; Santa Cruz Biotechnology, Inc.) and anti-GAPDH (sc-137179, dilution 1:1,000; Santa Cruz Biotechnology, Inc.) were used as the primary antibodies.

Determination of MICB levels in the culture supernatant. Secretion of MICB by PANC-1 cells cultured under various conditions was assessed by ELISA using commercially available kits (R\&D Systems, Minneapolis, MN, USA) and following the manufacturer's instructions.

Assessment of surface MICB expression. For the detection of membrane-bound MICB, cells were incubated with an anti-MICB-specific antibody (sc-80527, Santa Cruz Biotechnology, Inc.) and stained with phycoerythrin (PE)-goat anti-mouse immunoglobulin (BD Biosciences, Franklin Lakes, NJ, USA) as a secondary reagent and then subjected to flow cytometry. Flow cytometry was performed using a FACScan flow cytometer (Becton-Dickinson, San Jose, CA, USA).

3-(4,5-dimethylthiazol-2-yl)-2,5-diphenyltetrazolium bromide (MTT) assay. The viability of the PANC-1 cells was determined by MTT assay. Cells were stained with MTT (Amresco, Solon, OH, USA). Following $4 \mathrm{~h}$ of additional incubation, the medium was discarded, $100 \mu \mathrm{l}$ of acidic iso-propanol $(0.1 \mathrm{M}$ $\mathrm{HCl}$ in absolute isopropanol) was added and the plate was agitated gently. Absorbance was measured on an ELISA reader at a test wavelength of $540 \mathrm{~nm}$.

Statistical analysis. Data are reported as the means \pm standard deviation (SD), range and frequencies. MICB and ADAM15 expression for pancreatic cancer tissues and normal pancreatic tissue groups were compared using the Wilcoxon rank sum test. The correlation between the clinicopathological characteristics and the expressions of MICB and ADAM15 were analyzed using the Chi-square test. The correlations between MICB and ADAM15 were determined using the Spearman's rank correlation coefficient. Continuous variables were compared using the Student's t-test or ANOVA if normally distributed and the Wilcoxon rank sum test, if distributions were non-parametric. $\mathrm{P}<0.05$ (two-tailed) was considered to indicate a statistically significant difference. The calculations were performed using the SPSS 13.0 software (SPSS Inc., Chicago, IL, USA).

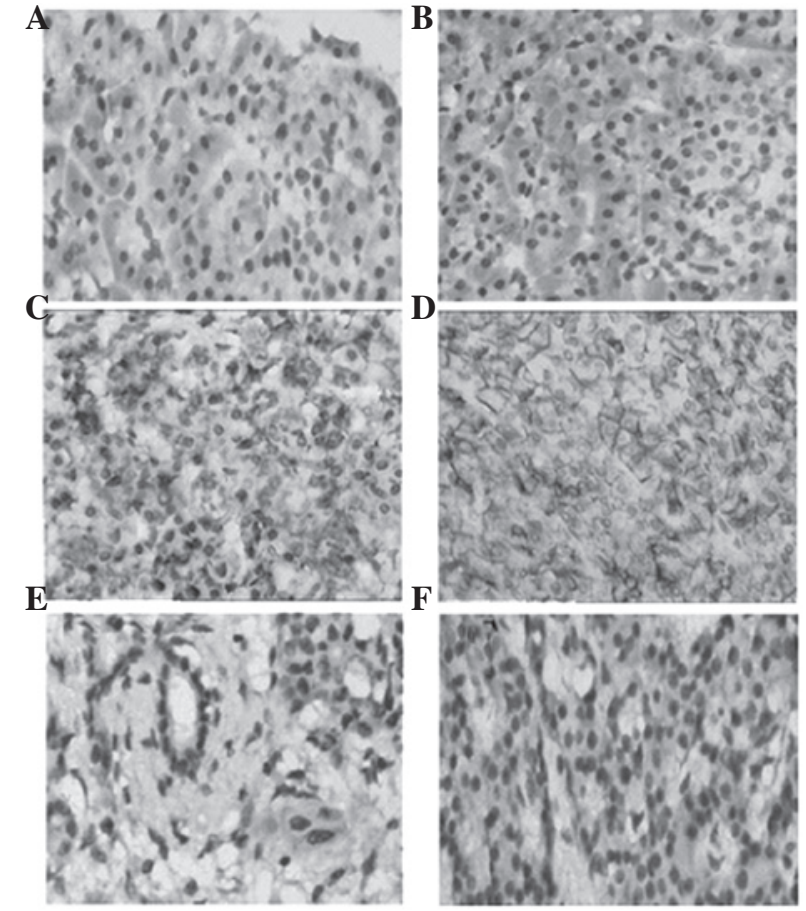

Figure 1. MICB and ADAM15 immunohistochemical staining (SABC method, magnification, $\mathrm{x} 400$ ). (A) Negative expression of MICB in normal pancreatic tissues. (B) Negative expression of ADAM15 in normal pancreatic tissues. (C) Negative membranous expression of MICB in pancreatic cancer tissues, positive staining for MICB was only diffusely located in the stroma in TNM stage IV. (D) Positive staining for MICB was localized on the membrane of tumor cells in carcinomas with TNM stage I. (E) Negative expression of ADAM15 in pancreatic cancer tissues with TNM stage I. (F) Strong expression of ADAM15 in pancreatic cancer tissues in TNM stage IV. MICB, MHC class I polypeptide-related sequence B.

\section{Results}

Expression levels of MICB and ADAM15 in pancreatic cancer and normal pancreatic tissues. The MICB expression was predominantly distributed on the membrane of the cells. The positive rates of MICB expression were 69.9\% (65/93) in pancreatic cancer tissues and 6.7\% (1/15) in normal pancreatic tissues. The Wilcoxon rank sum test indicated that the expression of MICB in pancreatic cancer was significantly higher compared with that in normal pancreatic tissues $(\mathrm{P}<0.01)$. Positive ADAM15 immunostaining was intensely located in cytoplasm and on the cell membrane. The positive rates of ADAM15 expression were $78.5 \%$ (73/93) in pancreatic cancer tissues and $13.3 \%(2 / 15)$ in normal tissues of the pancreas. The expression of ADAM15 in normal pancreatic tissues was significantly lower compared with that in pancreatic cancer tissues $(\mathrm{P}<0.01)$. The immunohistochemical staining results of MICB and ADAM15 are shown in Table I. The immunohistochemical staining is shown in Fig. 1.

Correlation between MICB, ADAM15 expression and clinicopathological characteristics. The correlation between the clinicopathological characteristics of pancreatic cancer patients and expression levels of MICB and ADAM15 is summarized in Table II. A significant difference was observed in the MICB expression with regard to the histological grade $(\mathrm{P}=0.033)$ and TNM stages $(\mathrm{P}=0.016)$. There was no significant correlation 
Table I. Expression of MICB and ADAM15 in pancreatic cancer and normal pancreatic tissues.

\begin{tabular}{|c|c|c|c|c|c|c|c|}
\hline \multirow[b]{2}{*}{ Groups } & \multirow[b]{2}{*}{ No. } & \multicolumn{3}{|c|}{ MICB } & \multicolumn{3}{|c|}{ ADAM15 } \\
\hline & & - & + & ++ & - & + & ++ \\
\hline Pancreatic cancer tissues & 93 & 28 & 39 & 26 & 20 & 28 & 45 \\
\hline Normal pancreatic tissues & 15 & 14 & 1 & 0 & 13 & 2 & 0 \\
\hline
\end{tabular}

-, no staining in cancer cells; +, the staining of the cancer cells is a little stronger compared with that of the cancer stroma in the whole area, or much stronger in a limited $(<20 \%)$ area; ++ , the staining of the cancer cells is much stronger compared with that of the cancer stroma in the whole section. MICB, MHC class I polypeptide-related sequence B.

Table II. Correlation between MICB and ADAM15 expression and clinicopathological characteristics.

\begin{tabular}{|c|c|c|c|c|c|c|c|}
\hline \multirow[b]{2}{*}{ Characteristics } & \multirow[b]{2}{*}{ No. } & \multicolumn{3}{|c|}{ MICB } & \multicolumn{3}{|c|}{ ADAM15 } \\
\hline & & High & Low & P-value & Positive & Negative & P-value \\
\hline \multicolumn{8}{|l|}{ Age (years) } \\
\hline$\leq 65$ & 50 & 18 & 32 & 0.062 & 41 & 9 & 0.375 \\
\hline$>65$ & 43 & 8 & 35 & & 32 & 11 & \\
\hline \multicolumn{8}{|l|}{ Gender } \\
\hline Male & 56 & 15 & 41 & 0.757 & 42 & 14 & 0.313 \\
\hline Female & 37 & 11 & 26 & & 31 & 6 & \\
\hline \multicolumn{8}{|l|}{ Tumor size $(\mathrm{cm})$} \\
\hline$\leq 3$ & 45 & 14 & 31 & 0.512 & 33 & 12 & 0.241 \\
\hline$>3$ & 48 & 12 & 36 & & 40 & 8 & \\
\hline \multicolumn{8}{|c|}{ Histological grade } \\
\hline Well & 28 & 13 & 15 & 0.033 & 21 & 7 & 0.658 \\
\hline Moderately & 39 & 8 & 31 & & 30 & 9 & \\
\hline Poorly & 26 & 5 & 21 & & 22 & 4 & \\
\hline \multicolumn{8}{|c|}{ Lymph node metastasis } \\
\hline Positive & 39 & 9 & 30 & 0.373 & 34 & 5 & 0.022 \\
\hline Negative & 24 & 8 & 16 & & 15 & 9 & \\
\hline \multicolumn{8}{|c|}{ Distant metastasis } \\
\hline Present & 14 & 6 & 8 & 0.305 & 9 & 5 & 0.293 \\
\hline Absent & 79 & 20 & 59 & & 64 & 15 & \\
\hline \multicolumn{8}{|l|}{ TNM stage } \\
\hline I+II & 61 & 22 & 39 & 0.016 & 44 & 17 & 0.039 \\
\hline III+IV & 32 & 4 & 28 & & 29 & 3 & \\
\hline
\end{tabular}

TNM stage, according to 2009 tumor-node-metastasis (TNM) classification of malignant tumors by the International Union Against Cancer. MICB, MHC class I polypeptide-related sequence B.

between the MICB expression and the clinicopathological characteristics with regard to age, gender, tumor size and distant or lymph node metastases ( $\mathrm{P}>0.05$, respectively). The ADAM15 expression was found to correlate with lymph node metastasis $(\mathrm{P}=0.022)$ and TNM stages $(\mathrm{P}=0.039)$. However, the ADAM15 expression was not associated with age, gender, tumor size, histological grade or distant metastasis $(\mathrm{P}>0.05)$.

Correlation between $M I C B$ and ADAM15 expression in pancreatic cancer tissues. The Spearman's rank test suggested that the expression of MICB was correlated inversely with that of ADAM15 in pancreatic cancer tissues $(\mathrm{r}=-0.253, \mathrm{P}=0.014$, Table III).

ADAM15 mediates the shedding of MICB in PANC-1 cells. To directly verify if ADAM15 is involved in MICB ectodomain shedding of PANC-1 cells, PANC-1 cells were transfected with siRNA targeted against endogenous human ADAM15. No difference was noted in proliferation between the control and ADAM15 knockdown cells (data not shown). 
Table III. Correlation between MICB and ADAM15 expression in pancreatic cancer.

\begin{tabular}{lrrrr}
\hline & \multicolumn{3}{c}{ ADAM15 } \\
\cline { 2 - 4 } MICB & - & + & ++ & Total \\
\hline- & 5 & 7 & 16 & 28 \\
+ & 4 & 13 & 22 & 39 \\
++ & 11 & 8 & 7 & 26 \\
Total & 20 & 28 & 45 & 93 \\
\hline
\end{tabular}

-, no staining in cancer cells; + , the staining of the cancer cells is a little stronger compared with that of the cancer stroma in the whole area, or much stronger in a limited $(<20 \%)$ area; ++ , the staining of the cancer cells is much stronger compared with that of the cancer stroma in the whole section. MICB, MHC class I polypeptide-related sequence $B$.

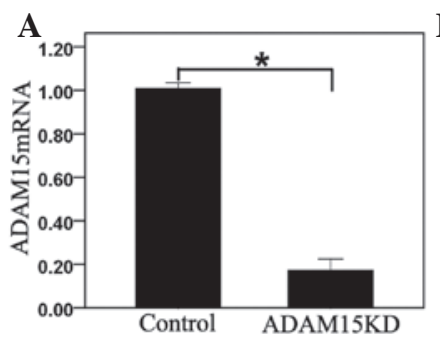

B
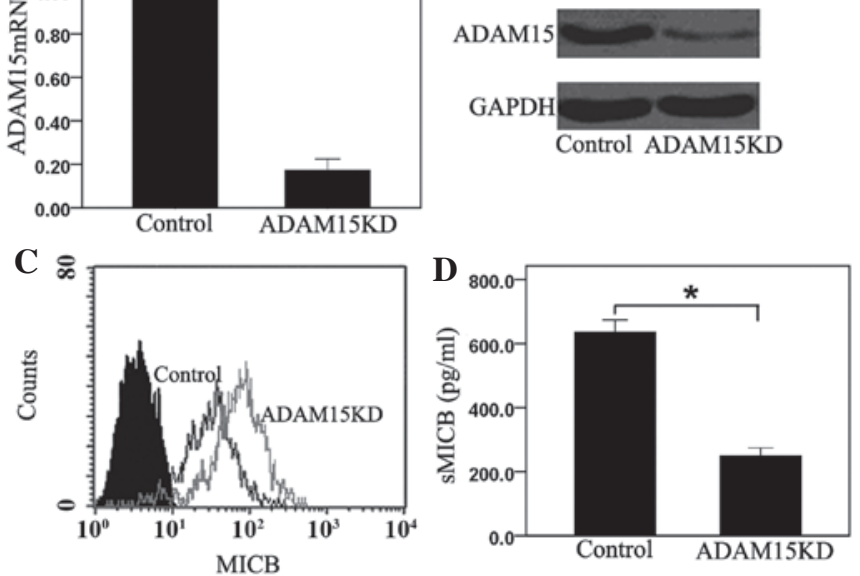

Figure 2. Expression of ADAM15 and MICB in ADAM15 knockdown PANC-1 cells. Cells were treated with ADAM15 siRNA (ADAM15KD) or control siRNA (Control) for $24 \mathrm{~h}$, and subjected to analysis of ADAM15 expression by (A) real-time RT-PCR and (B) western blotting. At the same time, the membrane-bound MICB and sMICB was evaluated by (C) flow cytometry and (D) ELISA. ${ }^{\mathrm{P}}<0.05$. MICB, MHC class I polypeptide-related sequence $B$.

The downregulation of ADAM15 transcripts and ADAM15 protein was monitored by real-time RT-PCR and by western blotting, respectively. The siRNA duplexes targeted against ADAM15 depleted endogenous levels of the mRNA by $85.88 \pm 2.73 \%$ (Fig. $2 \mathrm{~A}$ ) and the protein by $74.39 \pm 3.26 \%$, when compared with cells transfected with control siRNA (Fig. 2B). Knockdown of ADAM15 for PANC-1 cells resulted in a $40.74 \pm 3.15 \%$ increase in membrane-bound MICB (Fig. 2C) and in a $59.58 \pm 4.65 \%$ reduction of sMICB levels in the culture medium (Fig. 2D). Taken together, the results of the current study make a strong case that ADAM15 is a principal sheddase for membrane-bound MICB in PANC-1 cells.

Gemcitabine suppresses ADAM15 expression and inhibits $M I C B$ shedding of PANC-1 cells. The cytotoxicity of
A
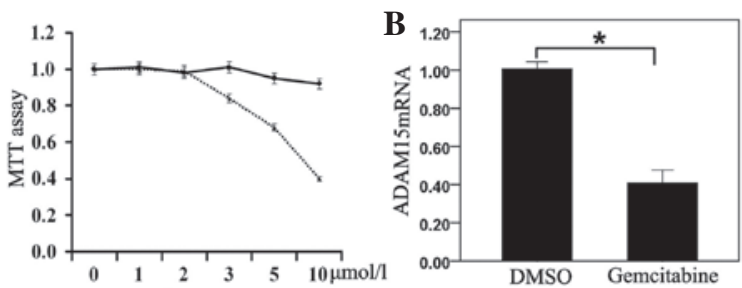

C
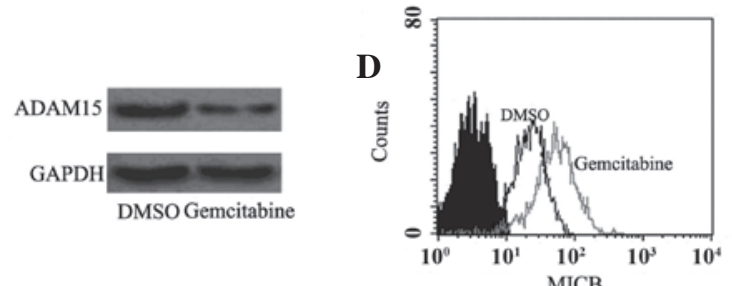

E
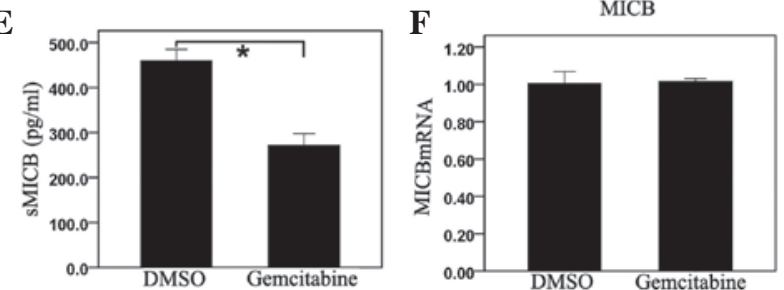

Figure 3. Changes of ADAM15 and MICB expression in PANC-1 cells after exposure to gemcitabine. (A) PANC-1 cells were treated with different concentrations of gemcitabine (dotted lines) or vehicle (DMSO; solid lines) for $24 \mathrm{~h}$, and the viability of the cells was evaluated by the MTT assay. Cells were treated with $0.5 \mu \mathrm{mol} / 1$ gemcitabine or vehicle (DMSO) for $24 \mathrm{~h}$ and ADAM15 expression was evaluated by (B) real-time RT-PCR and (C) western blotting. The membrane-bound MICB and SMICB expression was evaluated by (D) flow cytometry and (E) ELISA. (F) mRNA levels of MICB were evaluated by real-time RT-PCR. " $\mathrm{P}<0.05$. MICB, MHC class I polypeptide-related sequence $\mathrm{B}$; sMICB, soluble MICB.

gemcitabine to PANC-1 cells was evaluated using the MTT assay. Gemcitabine was found to have little effect on the growth of PANC-1 cells at concentrations of $\leq 2 \mu \mathrm{mol} / 1$ for $24 \mathrm{~h}$ (Fig. 3A). Based on this finding, gemcitabine with concentrations of $0.5 \mu \mathrm{mol} / 1$ was used to examine the biological effect on PANC-1 cells. PANC-1 cells were cultured for $24 \mathrm{~h}$ with gemcitabine and ADAM15 expression was evaluated at the mRNA and protein levels. Gemcitabine suppressed ADAM15 expression in PANC-1 cells (Fig. 3B and C). Treatment with gemcitabine was also observed to have markedly augmented membrane-bound MICB expression (Fig. 3D) and significantly decreased sMICB in PANC-1 cells (Fig. 3E). However, the mRNA levels of MICB did not change in gemcitabine-treated PANC-1 cells (Fig. 3F).

Gemcitabine inhibits MICB shedding of PANC-1 cells by suppressing ADAM15. To verify whether gemcitabine inhibits MICB ectodomain shedding through the suppression of ADAM15, PANC-1 cells were transfected with ADAM15 siRNA or control siRNA and then subjected to treatment with gemcitabine or DMSO. The data showed that gemcitabine upregulated MICB surface expression (Fig. 4A and C) and downregulated the levels of sMICB in control cells (Fig. 4D). Nevertheless, membrane-bound MICB (Fig. 4B and C) and sMICB levels (Fig. 4D) did not change in ADAM15 knockdown PANC-1 cells. These results suggest that gemcitabine inhibits MICB shedding in PANC-1 cells by downregulating the expression of ADAM15. 

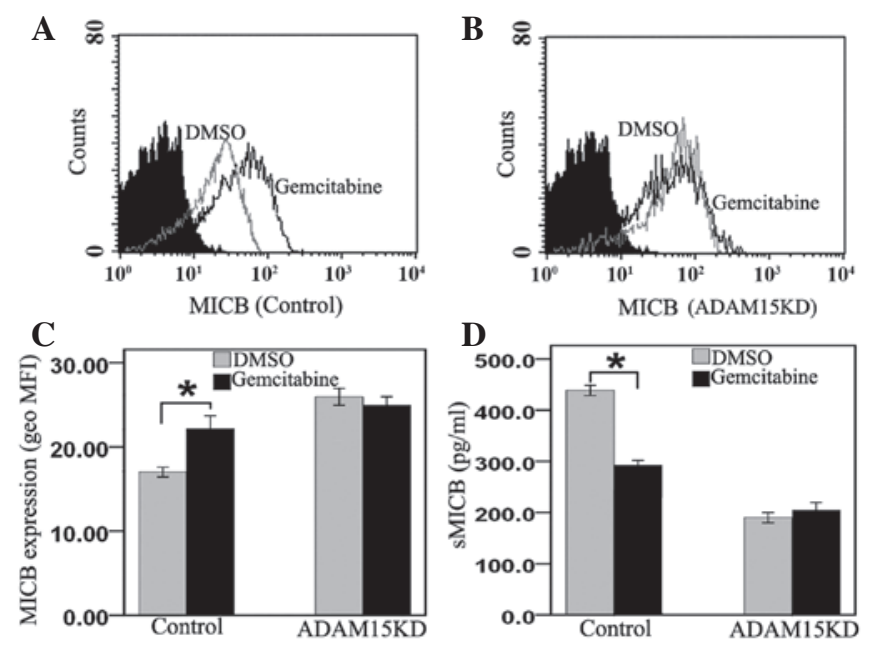

Figure 4 Gemcitabine-mediated shedding of MICB is ADAM15-dependent Cells were transfected with ADAM15 siRNA (ADAM15KD) or control siRNA (Control) for $24 \mathrm{~h}$ and then cultured with $0.5 \mu \mathrm{mol} / 1$ of gemcitabine or vehicle (DMSO) for $24 \mathrm{~h}$. (A and B) The expression of membrane-bound MICB was evaluated by flow cytometry and $(\mathrm{C})$ the statistical analysis shown as the median of geometric mean fluorescence intensity (geo MFI) of MICB is presented. (D) sMICB in the culture supernatant was evaluated by ELISA ${ }^{*} \mathrm{P}<0.05$. MICB, MHC class I polypeptide-related sequence B; sMICB, soluble MICB.

\section{Discussion}

Although upregulation of MICB had been observed in pancreatic cancer cell lines, information on MICB expression on human pancreatic cancer is scarce (10). In the present study, the expression of MICB was examined in 93 pancreatic cancer patients using immunohistochemistry. MICB was found to be overexpressed in PDAC compared with normal pancreatic tissues. Thus, MICB is hypothesized to be associated with the malignant transformation of pancreatic cancer. Moreover, a significant difference was noted in the MICB expression with regard to the histological grade and TNM stages. The data also showed that although it was highly expressed in pancreatic cancer, membrane-bound MICB was prevalent only in low-grade cancers and positive staining for MICB was diffusely distributed in the stroma in high-grade cancers. In particular, in carcinomas with TNM stage IV, most tumor cells showed negative cell surface MICB immunoreactivity and diffuse MICB staining was shown in the stroma. To the best of our knowledge, this is the first study demonstrating the correlation between MICB expression and clinicopathological characteristics in pancreatic cancer. MICB expressed on the surface of pancreatic cancer cells may be released into the tumor stroma and circulation in high-grade cancers (21). Serum levels of sMICB in pancreatic cancer patients were found to correlate significantly with high tumor stage and poor differentiation (11). MICB shedding is thought to be a principal mechanism by which tumor cells escape from NKG2D-mediated immunosurveillance in pancreatic cancer (22). Therefore, it is necessary to elucidate the molecular mechanisms of MICB ectodomain shedding in pancreatic cancer.

ADAM15, cloned from mammary epithelial cells, is a catalytically active member of the ADAM family of disinte- grin proteinases (23). One of the major function of ADAM15 is the proteolytic release of ectodomains of transmembranous proteins, including cytokines, growth factors and cell adhesion molecules, from the cell surface (22). ADAM15 has been reported to be frequently overexpressed in tumors and is thought to play key roles in various steps of tumorigenesis (19). To date, limited data have been published on the expression of ADAM15 in pancreatic cancer. The present study demonstrated that ADAM15 expression is significantly higher in PDAC compared with normal pancreatic tissues. The results are consistent with findings in various additional malignant tumors, including stomach and colorectal tumors (24). In this study, ADAM15 expression was closely correlated with lymph node metastasis and TNM stages, suggesting that ADAM15 may contribute to the invasive growth and progression of pancreatic cancer.

A positive correlation between MICB and ADAM15 expression has also been confirmed in the present study. Of note, the expression of membrane-bound MICB was inversely correlated with that of ADAM15 in pancreatic cancer tissues. With the progression of pancreatic cancer, the expression of ADAM15 increased, but MICB was released into the tumor stroma and bloodstream as SMICB (21). To the best of our knowledge, this is the first study to report a large number of clinical samples showing the exact correlation between MICB and ADAM15.

The finding that ADAM15 was essential for the cleavage of MICB was supported by testing the effect of the siRNA-mediated knockdown of ADAM15 on the release of SMICB. In this study, we demonstrated that ADAM15 knockdown resulted in increased membrane-bound MICB and decreased sMICB. The present study is the first to demonstrate that ADAM15 is involved in the shedding of the MICB in PANC-1 cells. The observations in this study suggest that ADAM15 may be a potential therapeutic target for inhibiting MICB shedding.

Gemcitabine is currently the standard chemotherapy for unresectable pancreatic cancer (25). Gemcitabine is a nucleoside analog that exerts its antitumor activity via multiple mechanisms of action. These include i) incorporation of gemcitabine into replicating DNA, which inhibits DNA replication and cell growth; ii) masked DNA chain termination; iii) several self-potentiation mechanisms that serve to increase intracellular levels of the active compound. It thus halts DNA synthesis and is invisible to DNA repair systems, leading the cells into the apoptotic pathway (26). Despite the promising results associated with gemcitabine, its mode of action and of the enzymes it interacts with have yet to be fully documented (27). Our results demonstrated that gemcitabine downregulated ADAM15 expression, thereby resulting in the increased expression of membrane-bound MICB and the decreased production of sMICB. However, the data showed that the mRNA levels of MICB did not change following exposure to gemcitabine in PANC-1 cells. To the best of our knowledge, we are the first to report that gemcitabine is able to modulate PANC-1 cells by downregulating ADAM15 expression, thereby inhibiting MICB ectodomain shedding. The combination of molecularly targeted therapy and immunotherapy targeting activation of NK cells may improve the antitumor effect against unresectable pancreatic cancer and the prognosis of patients with pancreatic cancer (28). Further 
studies (e.g., animal models) are required to establish whether gemcitabine results in reduced sMICB levels and an enhanced NKG2D-mediated tumor elimination.

In conclusion, although the expression of surface MICB at an early stage of pancreatic cancer is thought to lead to the activation of effector cells and the destruction of the tumor cells, the development of pancreatic cancer may lead to the shedding of MICB. The present study has demonstrated unequivocally for the first time that MICB is constitutively shed by ADAM15. We have also shown that gemcitabine suppressed MICB ectodomain shedding from PANC-1 cells through the inhibition of ADAM15. The present study sheds light on previously unrecognized effects of gemcitabine on modulating ADAM15 and MICB shedding, thus suggesting its use in chemoimmunotherapy against human pancreatic cancer.

\section{References}

1. Sargent M, Boeck S, Heinemann V, Jauch KW, Seufferlein T and Bruns CJ: Surgical treatment concepts for patients with pancreatic cancer in Germany - results from a national survey conducted among members of the 'Chirurgische Arbeitsgemeinschaft Onkologie' (CAO) and the 'Arbeitsgemeinschaft Internistische Onkologie' (AIO) of the Germany Cancer Society (DKG). Langenbecks Arch Surg 396: 223-229, 2011.

2. Hackert T, Büchler MW and Werner J: Surgical options in the management of pancreatic cancer. Minerva Chir 64: 465-476, 2009.

3. Siegel R, Desantis C, Virgo K, et al: Cancer treatment and survivorship statistics, 2012. CA Cancer J Clin 62: 220-241, 2012.

4. Levi F, Lucchini F, Negri E and La Vecchia C: Pancreatic cancer mortality in Europe: the leveling of an epidemic. Pancreas 27: 139-142, 2003

5. Waldmann TA: Immunotherapy: past, present and future. Nat Med 9: 269-277, 2003.

6. Dranoff G: Coordinated tumor immunity. J Clin Invest 111: 1116$1118,2003$.

7. Groh V, Rhinehart R, Secrist H, Bauer S, Grabstein KH and Spies T: Broad tumor-associated expression and recognition by tumor-derived gamma delta T cells of MICB and MICB. Proc Natl Acad Sci USA 96: 6879-6884, 1999.

8. González S, Groh V and Spies T: Immunobiology of human NKG2D and its ligands. Curr Top Microbiol Immunol 298: 121-138, 2006.

9. Wu JD, Higgins LM, Steinle A, Cosman D, Haugk K and Plymate SR: Prevalent expression of the immunostimulatory MHC class I chain-related molecule is counteracted by shedding in prostate cancer. J Clin Invest 114: 560-568, 2004.

10. Ohashi M, Yoshida K, Kushida M, et al: Adenovirus-mediated interferon gene transfer induces regional direct cytotoxicity and possible systemic immunity against pancreatic cancer. $\mathrm{Br} \mathrm{J}$ Cancer 93: 441-449, 2005.
11. Märten A, von Lilienfeld-Toal M, Büchler MW and Schmidt J: Soluble MIC is elevated in the serum of patients with pancreatic carcinoma diminishing gammadelta $\mathrm{T}$ cell cytotoxicity. Int J Cancer 119: 2359-2365, 2006.

12. Diefenbach A, Jensen ER, Jamieson AM and Raulet DH: Rael and H60 ligands of the NKG2D receptor stimulate tumour immunity. Nature 413: 165-171, 2001.

13. Groh V, Wu J, Yee C and Spies T: Tumour-derived soluble MIC ligands impair expression of NKG2D and T-cell activation. Nature 419: 734-738, 2002.

14. Salih HR, Goehlsdorf D and Steinle A: Release of MICB molecules by tumor cells: mechanism and soluble MICB in sera of cancer patients. Hum Immunol 67: 188-195, 2006.

15. Boutet $\mathrm{P}$, Agüera-González S, Atkinson S, et al: Cutting edge: the metalloproteinase ADAM17/TNF-alpha-converting enzyme regulates proteolytic shedding of the MHC class I-related chain B protein. J Immunol 182: 49-53, 2009.

16. Hidalgo M: Pancreatic cancer. N Engl J Med 362: 1605-1617, 2010.

17. Plate JM, Plate AE, Shott S, Bograd S and Harris JE: Effect of gemcitabine on immune cells in subjects with adenocarcinoma of the pancreas. Cancer Immunol Immunother 54: 915-925, 2005.

18. Li K, Mandai M, Hamanishi J, et al: Clinical significance of the NKG2D ligands, MICA/B and ULBP2 in ovarian cancer: high expression of ULBP2 is an indicator of poor prognosis. Cancer Immunol Immunother 58: 641-652, 2009.

19. Schütz A, Härtig W, Wobus M, Grosche J, Wittekind Ch and Aust G: Expression of ADAM15 in lung carcinomas. Virchows Arch 446: 421-429, 2005.

20. Chen X, Liao J, Lu Y, Duan X and Sun W: Activation of the PI3K/ Akt pathway mediates bone morphogenetic protein 2-induced invasion of pancreatic cancer cells PANC-1. Pathol Oncol Res 17: 257-261, 2011.

21. Holdenrieder S, Stieber P, Peterfi A, Nagel D, Steinle A and Salih HR: Soluble MICB in malignant diseases: analysis of diagnostic significance and correlation with soluble MICA. Cancer Immunol Immunother 55: 1584-1589, 2006.

22. Kaiser BK, Yim D, Chow IT, et al: Disulphide-isomerase-enabled shedding of tumour-associated NKG2D ligands. Nature 447: 482-486, 2007.

23. Najy AJ, Day KC and Day ML: The ectodomain shedding of E-cadherin by ADAM15 supports ErbB receptor activation. J Biol Chem 283: 18393-18401, 2008.

24. Lucas N, Najy AJ and Day ML: The therapeutic potential of ADAM15. Curr Pharm Des 15: 2311-2318, 2009.

25. Heinemann V, Boeck S, Hinke A, Labianca R and Louvet C: Meta-analysis of randomized trials: evaluation of benefit from gemcitabine-based combination chemotherapy applied in advanced pancreatic cancer. BMC Cancer 8: 82, 2008.

26. Abou-Alfa GK, Letourneau R, Harker G, et al: Randomized phase III study of exatecan and gemcitabine compared with gemcitabine alone in untreated advanced pancreatic cancer. J Clin Oncol 24: 4441-4447, 2006.

27. Yanagimoto H, Shiomi H, Satoi S, et al: A phase II study of personalized peptide vaccination combined with gemcitabine for non-resectable pancreatic cancer patients. Oncol Rep 24: 795-801, 2010.

28. Koido S, Homma S, Takahara A, et al: Current immunotherapeutic approaches in pancreatic cancer. Clin Dev Immunol 2011: 267539, 2011. 